\title{
Aspects linguistiques et cognitifs en jeu lors du traitement d'expressions démonstratives anaphoriques
}

\author{
Marion Fossard \\ Programme de maîtrise en orthophonie - Faculté de médecine - Université Laval, Québec (Canada). Centre de \\ recherche Université Laval Robert Giffard - Axe recherche évaluative et clinique \\ marion.fossard@rea.ulaval.ca
}

\section{Introduction}

Un des aspects les plus importants de la compréhension (mais aussi de la production) du langage concerne notre capacité à connecter, relier, organiser les différentes propositions discursives entre elles afin d'établir une représentation mentale cohérente du discours en cours de construction. Une façon essentielle d'établir cette cohérence passe par l'utilisation de différents types d'expressions référentielles, telles que les pronoms (il / elle...), les démonstratifs (celui-ci, ce garçon, cette fille ...), les descriptions définies (le garçon, la fille...) dont l'usage permet de maintenir la référence à des entités de discours préalablement mentionnées ou évoquées (Charolles, 1995; Cornish, 1999, 2001; Garnham, 2000 ; Gernsbacher \& Givon, 1995; Johnson-Laird, 1983; Sanford \& Garrod, 1989).

Ces différentes expressions, cependant, ne sont pas équivalentes en termes d'instructions de traitement qu'elles véhiculent, et le fait qu'elles ne soient pas facilement 'interchangeables' les unes avec les autres a donné lieu, ces dernières années, à un grand nombre de travaux visant à repérer les relations existant entre marqueurs de référence (pronoms, etc...) et état d'activation ou d'accessibilité qu'un référent donné est supposé avoir dans la représentation ou le modèle de discours en cours (accessibilité élevée ou 'en focus' pour un référent très saillant - ex : le personnage principal d'une histoire ; accessibilité moyenne ou 'familière' pour un référent moins saillant - ex : le personnage secondaire ; accessibilité basse pour un nouveau référent - ex : apparition d'un nouveau personnage) (Ariel, 1990, 1996, 2006; Gundel, Hedberg, \& Zacharski, 1993, 2000; Strauss, 2002).

Différents modèles comme 'l'échelle d'accessibilité' (Accessibility marking scale) d'Ariel (1990, 1996, 2006) ou la 'hiérarchie du donné' (Givenness Hierarchy) de Gundel et al. (1993, 2000) suggèrent ainsi que les différentes expressions référentielles, de par leur sens spécifique servant à marquer le niveau d'accessibilité (élevée, moyenne, basse) ou le statut cognitif du référent attendu ('en focus', 'familier'...), signaleraient différentes façons selon lesquelles un énoncé peut - ou devrait - être résolu.

De fait, deux questions centrales se posent aux psycholinguistes :

1) en quoi les spécificités inhérentes à chaque type d'expression semblent-elles guider le traitement de l'énoncé de différentes façons?

2) comment le choix d'une expression (plutôt qu'une autre) affecte-t-il la manière selon laquelle l'auditeur ou le lecteur construit son modèle de discours?

Un grand nombre de travaux - essentiellement en anglais - ont été menés pour tenter d'apporter des éléments de réponses à ces questions. Cependant, force est de constater que très peu de types d'expressions ont été expérimentalement testés, la majorité des études s'étant essentiellement focalisée sur le seul contraste existant entre pronom anaphorique il / elle et expressions pleines (ex : descriptions 
définies) - réduites le plus souvent à la simple répétition de nom - (Greene, McKoon \& Ratcliff., 1992 ; Garrod, Freudenthal \& Boyle., 1994 ; Hudson-D’Zmura \& Tanenhaus., 1998 ; Fossard, 1999, pour des résultats en français).

Le présent projet vise à analyser le comportement sémantico-référentiel d'expressions démonstratives sur les processus de traitement de phrases en contexte discursif et à étudier leur impact sur la cohérence du discours. Ces expressions, bien décrites d'un point de vue linguistique (Cornish, 1999, 2001; De Mulder, 1997, 2000; Kleiber, 2001) n'ont jusqu'à présent fait l'objet que de très peu de travaux psycholinguistiques (mais voir l'étude de Brown-Schmidt, Byron \& Tanenhaus 2005 en anglais; celle de Fossard \& Rigalleau, 2005 en français, et celle de Fossard (2006) pour une comparaison franco-anglaise). Ceci est d'autant plus surprenant que leur nature déictique (i.e., le fait qu'elles permettent d'attirer l'attention sur un référent particulier: objet, individu...) en fait des expressions très originales pour l'étude du traitement anaphorique en général, et pour l'étude des processus interprétatifs mis en œuvre par des lecteurs lors de la résolution référentielle en particulier.

Mieux comprendre la distribution et l'interprétation des formes démonstratives dans le langage constitue une étape de plus dans la compréhension du fonctionnement des systèmes anaphoriques. Les retombées de ce travail pourront intéresser tout autant le domaine de la didactique des langues que celui des troubles discursifs en pathologie du langage.

\section{Problématique}

Inscrivant notre travail dans une approche cognitive ou procédurale des marqueurs de référence, nous avons étudié lors de travaux pionniers réalisés dans le cadre du doctorat (travaux en langue française) et du post-doctorat (travaux en langue anglaise) la possibilité qu'offriraient les démonstratifs d'entraîner un changement référentiel dans l'organisation du discours en cours ou, en termes plus cognitifs, un « changement de focus » (cf. Gundel, 1998).

Les expériences réalisées alors portaient sur la résolution anaphorique de démonstratifs, c'est-à-dire sur des démonstratifs présupposant l'existence préalable d'un cadre de référence à l'intérieur duquel le référent attendu est déjà connu (cf. Cornish, 1999). Utilisant un paradigme d'enregistrement de temps de lecture nous avons investigué dans deux séries d'expérience, l'une portant sur le pronom démonstratif français celui-ci / celle-ci, et l'autre portant sur l'expression démonstrative anglaise that $\mathrm{N}$ (déterminant démonstratif that suivi d'un nom tête man/woman, en l'occurence), les conséquences de la valeur procédurale de ces marqueurs sur le traitement cognitif en jeu en analysant leur fonctionnement en contraste avec celui d'une expression prototypiquement anaphorique : le pronom anaphorique de troisième personne (il / elle en français, et he /she en anglais).

L'étude de ces contrastes nous a semblé intéressante pour mettre en évidence les propriétés procédurales des démonstratifs. En effet, contrairement au pronom anaphorique (il / elle - he / she) dont l'usage est réservé aux cas où le référent ciblé est supposé être très accessible, 'en focus' dans la représentation de discours en cours (Gordon, Grosz, \& Gilliom, 1993 ; Gundel, 1998 ; Sanford, Moar \& Garrod, 1988 ; Walker, Joshi \& Prince, 1998 ...), les démonstratifs (pronom ou déterminant) pourraient servir - du fait de la procédure déictique qui les caractérise - à réorienter le discours en dirigeant l'attention de l'interlocuteur sur un référent qui n'est pas le plus fortement activé (ou en focus).

Les résultats obtenus ont confirmé nos hypothèses. Indicateur d'accessibilité élevée, le pronom anaphorique agit bien comme un marqueur privilégié d'accès à l'entité la plus focalisée dans la représentation de discours (avec l'obtention de temps de lecture plus rapides lorsque le pronom anaphorique $i l /$ elle réfère à l'entité focalisée plutôt qu'à l'entité moins focalisée). En revanche, du fait de la saisie déictique qu'ils opèrent sur le référent, les démonstratifs, marqueurs d'accessibilité 'intermédiaire' (Ariel, 1990, 1996, 2006) ou de 'focus moyen' (Strauss, 2002) s'avèrent être de très 'bons 
outils' référentiels pour orienter l'attention du lecteur sur un référent moins focalisé, moins accessible temps de lecture plus rapides lorsque le démonstratif réfère à l'entité la moins focalisée plutôt qu'à l'entité la plus focalisée.

L'accessibilité ou le statut cognitif des référents apparait ainsi comme une contrainte forte s'exerçant sur le traitement des expressions référentielles. Une contrainte forte, certainement, mais qui n'apporte peutêtre pas une réponse totalement satisfaisante - ou suffisante - au problème posé par l'interprétation des marqueurs de référence.

Dénoncées par un certain nombre de linguistes, parmi lesquels Cornish, 1999, 2001; DeMulder, 2000; Kleiber, 1994, 2001; Maes \& Noordman, 1995..., les théories cognitives uniquement basées sur l'accessibilité ou le statut cognitif que le référent ciblé est supposé avoir dans le modèle de discours en cours manqueraient à voir que le sens des marqueurs de référence n'est pas réductible au seul statut cognitif du référent visé.

C'est en effet le reproche majeur que ces linguistes adressent aux modèles d'accessibilité cognitive, lesquels, finalement, n'envisagent les différentes expressions référentielles qu'en termes " identificationnels » ou "identificatoires » (Kleiber, 2001), considérant seulement leur potentiel à identifier, à accéder à des référents de discours possédant divers degrés d'accessibilité.

En conséquence, ces modèles ne portent attention qu'à la phrase ou les phrases antérieures pour sélectionner le référent-candidat, et la prédication anaphorique : la phrase-hote, celle qui héberge le marqueur référentiel, est reléguée au second plan. Celle-ci n'est alors considérée que comme simple phrase de 'contrôle' destinée seulement à confirmer la sélection opérée, et de fait, elle n'intervient pas positivement dans le processus interprétatif.

Or, tel que suggéré par DeMulder (2000) ou Kleiber (2001), ce ne serait pas tant l'accessibilité per se des référents qui serait en jeu dans le traitement des expressions référentielles que leur statut par rapport à la continuité discursive, ce qui pourrait être particulièrement important à prendre en compte pour l'étude des démonstratifs. En effet, les descriptions démonstratives (constituées d'un déterminant démonstratif ce suivi d'un nom tête, ex : garçon / fille...) ne font pas simplement qu'identifier ou accéder à une représentation de discours particulière (i.e., un référent), elles la modifient (cf. Maes \& Noordman, 1995).

De fait, pour les modèles basés uniquement sur l'accessibilité des référents de discours, les démonstratifs 'posent problème'. Ne prenant pas en compte la modification qu'introduit l'usage de la description démonstrative sur la représentation de discours en cours, ou - dit autrement - ne prenant pas en compte la rupture qu'entraîne l'usage de la description démonstrative par rapport à la situation dans laquelle le référent était précédemment impliqué (cf. Kleiber, 2001), ces modèles manquent à voir que l'accessibilité 'intermédiaire' ou le 'focus moyen' que marque la description démonstrative n'est peut-être qu'une conséquence d'un sens de base plus profond des démonstratifs, lié à leur valeur " token-réflexive », c'està-dire au fait qu'ils exigent d'identifier le référent à partir de leur occurrence, leur token même (DeMulder, 1997, 2000; Kleiber, 1986).

Cette exigence token-réflexive aurait comme conséquence le fait que les descriptions démonstratives, en rompant avec les événements en cours, introduisent de la discontinuité dans le discours. En cela, leur interprétation contrasterait avec celle d'un pronom anaphorique de troisième personne (il / elle) ou avec celle d'une description définie (le garçon / la fille...), lesquels, parce qu'ils saisissent le référent dans le prolongement de la situation antérieure 'manifeste' convoient davantage la continuité référentielle et attentionnelle (Kleiber, 1994, 2001). 


\section{Objectif et hypothèses}

Notre objectif principal est de tester l'hypothèse générale selon laquelle les descriptions démonstratives, lorsqu'elles sont utilisées anaphoriquement, sont spécialisées pour signaler une nouvelle orientation à l'intérieur du discours. De fait, lorsque le référent a déjà été présenté dans le discours, sa récupération par une description démonstrative doit impliquer quelque chose de plus que le simple maintien référentiel : elle doit impliquer une rupture par rapport au contexte de discours précédent.

- Ce faisant, nous testerons dans une première série d'études l'hypothèse plus spécifique selon laquelle les descriptions démonstratives, en tant qu'elles signalent une nouvelle orientation discursive, permettent d'attirer l'attention du lecteur sur un référent qui, bien que déjà introduit dans le discours, n'est cependant pas celui qui était attendu pour assurer la continuité référentielle. En d'autres termes, nous nous intéresserons à la possibilité qu'offre le démonstratif d'orienter l'attention sur un référent de discours peu proéminent, c'est-à-dire moins accessible, introduisant de fait une certaine discontinuité dans le discours.

Cette première hypothèse est assez proche de la vision que proposent les modèles d'accessibilité cognitive du fonctionnement des démonstratifs auxquels ils associent typiquement le statut cognitif de 'familier'. Cependant, contrairement à ces modèles, nous pensons que le statut cognitif de 'familier' que peut coder la description démonstrative n'est pas tant un trait définitoire de ce marqueur qu'un effet consécutif de son sens token-réflexif (voir deuxième série d'études ci-après).

- C'est en effet ce que nous souhaitons plus particulièrement tester dans la deuxième série d'études en posant comme deuxième hypothèse qu'une autre propriété des descriptions démonstratives est d'introduire un nouveau point de vue sur un référent déjà 'en focus', déjà très accessible.

Cette seconde hypothèse, incompatible avec les modèles d'accessibilité cognitive, est en revanche totalement compatible avec le sens de base token-réflexif des descriptions démonstratives mis au jour par les linguistes. En effet, un autre effet de ce sens de base (autre que celui d'attirer l'attention du lecteur sur un référent peu focalisé - cf. hypothèse 1-) serait d'introduire une nouvelle orientation discursive en présentant le référent déjà 'en focus', déjà très accessible, non pas dans le prolongement de la situation antérieure manifeste - en continuité avec le contexte de discours précédent - mais au contraire avec un changement de perspective, instaurant, de fait, un nouveau point de vue sur le référent.

\section{Première série d'études : méthodologie et approche proposée}

Dans le cadre de cette première série d'études, le fonctionnement référentiel de la description démonstrative : ce suivi d'un nom tête - fille /garçon - sera étudié en contraste avec celui de deux autres marqueurs : le pronom anaphorique il / elle et la description définie la fille /le garçon. Notre hypothèse est que, contrairement à ces deux marqueurs, la description démonstrative, de part la rupture qu'elle instaure par rapport au contexte de discours précédent, devrait être un bon outil référentiel pour attirer l'attention du lecteur sur un référent peu saillant, moins accessible. Pour chacun de ces 2 contrastes trois tâches seront utilisées : une tâche de jugement d'acceptabilité des textes et d'identification du référent ; une tâche de continuation de fragments de textes; et une tâche d'enregistrement de temps de lecture sur ordinateur.

\subsection{Matériel expérimental}

Le matériel expérimental est composé de vingt-quatre textes expérimentaux et quarante textes de remplissage (textes destinés à masquer la saillance des manipulations expérimentales). Les textes expérimentaux, composés de trois phrases chacun, sont tous bâtis sur le patron suivant (cf. exemple cidessous, tableau 1). 
Il s'agit de mini-scénarios mettant en scène deux personnages (phrases 1 et 2 , correspondant au contexte discursif). Le premier personnage ('Alice', dans l'exemple ci-dessous) correspond au protagoniste principal de la situation décrite, c'est-à -dire à l'entité la plus focalisée (Entité 1). Ce personnage est introduit en position de topique et il est ré-évoqué plusieurs fois dans le texte via différents pronoms. Le second personnage correspond à l'entité 2 , c'est-à -dire à l'entité la moins focalisée ('une serveuse', dans l'exemple donné ci-dessous). En effet, il apparaît en position objet via une description de rôle dans la scène, et contrairement à l'entité 1 , ce personnage n'est mentionné qu'une seule fois dans le texte et il est codé par un syntagme nominal indéfini. La troisième phrase du texte correspond à la phrase cible (celle sur laquelle on analysera les temps de lecture pour la tâche d'enregistrement des temps de lecture), c'est-à -dire à la phrase obtenue en croisant les deux facteurs de l'étude :

- $\quad$ Facteur 1 : type entité, déterminé sémantiquement par le verbe utilisé dans la phrase cible et qui oriente le traitement soit vers l'entité 1 (ex : commander) soit vers l'entité 2 (ex : conseiller)

- $\quad$ Facteur 2 : type marqueur référentiel, correspondant à la forme de l'expression référentielle utilisée dans la phrase cible pour référer à l'une des deux entités (il \elle vs. ce garçon \cette fille pour le premier contraste; et le garçon \la fille vs. ce garçon \cette fille pour le deuxième contraste)

Concernant spécifiquement la tâche d'enregistrement de temps de lecture, les textes sont présentés de façon segmentée à l'écran (indiqué par les doubles slashs (//) dans l'exemple). Une question (oui/non) portant sur la compréhension de la phrase cible est posée après la lecture de chaque texte.

Tableau 1 : Exemple d'un texte expérimental (illustrant le premier contraste) selon les quatre conditions expérimentales

\begin{tabular}{|c|c|}
\hline $\begin{array}{l}\text { Phrases } 1 \text { et } 2: \\
\text { (contexte discursif) }\end{array}$ & $\begin{array}{l}\text { Au restaurant, Alice }{ }_{(\text {entité 1) }} \text { adore prendre son temps pour lire le menu. // } \\
\text { La dernière fois, elle avait tellement hésité entre deux plats qu'elle avait } \\
\text { finalement demandé à une serveuse } \text { (entité 2) }_{\text {de l'aider à choisir quelque }} \\
\text { chose sur le menu. // }\end{array}$ \\
\hline \multicolumn{2}{|l|}{ Phrase 3 (phrase cible) } \\
\hline Entité $1 *$ pronom & En fait, elle a simplement commandé le pat de jour // \\
\hline Question (oui/non) & Est-ce qu'Alice a choisi le plat le plus dispendieux? \\
\hline Entité $1 * \mathrm{Ce} \mathrm{N}$ & En fait, cette fille a simplement commandé le plat du jour // \\
\hline Question (oui / non) & Est-ce qu'Alice a choisi le plat le plus dispendieux? \\
\hline Entité $2 *$ pronom & En fait, elle a simplement conseillé le plat du jour // \\
\hline Question (oui / non) & Est-ce que la serveuse a recommandé le plat le plus dispendieux? \\
\hline Entité $2 * \mathrm{Ce} \mathrm{N}$ & En fait, cette fille a simplement conseillé le plat du jour // \\
\hline Question (oui/non) & Est-ce que la serveuse a recommandé le plat le plus dispendieux? \\
\hline
\end{tabular}

Pour chaque tâche (tâche de jugement d'acceptabilité des textes et d'identification du référent ; tâche de continuation de fragments de textes; et tâche d'enregistrement de temps de lecture sur ordinateur), le plan expérimental utilisé sera un plan à croisement des textes avec les conditions. Ce plan permet que chacun 
des vingt-quatre textes expérimentaux rencontre les quatre conditions du croisement et qu'un participant donné ne lise chaque texte qu'une seule fois et lise autant de textes dans chacune des quatre conditions. Pour chaque participant, l'ordre des textes sera présenté de façon aléatoire.

\subsection{Procédure pour la tâche de jugement d'acceptabilité et d'identification du référent}

Cette tâche 'papier-crayon' a un double objectif. D'une part, elle permet d'estimer l'acceptabilité (jugement de cohérence) des textes dans leurs différentes conditions en utilisant une échelle à 7 points ( $1=$ très cohérent ; $7=$ pas cohérent du tout), et d'autre part, elle permet de s'assurer que l'identification du référent est bonne dans toutes les conditions ( $>90 \%$ ) en demandant aux participants d'entourer le nom du personnage correspondant au marqueur référentiel utilisé dans la phrase cible.

\subsection{Procédure pour la tâche de continuation de fragments de texte}

Cette autre tâche 'papier-crayon' a pour objectif de donner une mesure de la sensibilité des différentes expressions référentielles à l'accessibilité des référents en établissant quel personnage (Entité 1 ou Entité 2) les participants choisissent préférentiellement comme référent. Pour ce faire, les phrases cibles seront présentées de façon incomplète (ex : En fait, cette fille........) et les participants devront écrire une continuation en lien avec le texte présenté.

\subsection{Procédure pour la tâche d'enregistrement de temps de lecture}

Dans cette tâche, les phrases des textes apparaîtront de façon segmentée (indiqué par // dans l'exemple donné dans le tableau 1) sur un écran d'ordinateur (utilisation du logiciel E-Prime). La consigne indiquera aux participants de lire les textes segments par segments, le plus rapidement et le plus exactement possible. Après lecture de chaque segment, les participants devront appuyer sur le bouton désigné de la boîte à boutons placée devant eux, ce qui effacera le segment lu de l'écran et le remplacera par le segment suivant, et ainsi de suite jusqu'à la fin du texte.

Les temps de lecture (TL) des phrases cibles seront enregistrés pour chaque participant. Une seconde après la fin de la présentation de la phrase cible, la question (oui/non) apparaîtra. Les participants devront y répondre le plus rapidement et le plus exactement possible en appuyant sur le bouton désigné de la boîte à boutons.

En accord avec notre hypothèse, nous formulons les prédictions suivantes :

Pour les contrastes 1 et 2 : les temps de lecture des phrases cibles contenant une description démonstrative seront plus rapides lorsque la description démonstrative réfère à l'entité 2 plutôt qu'à l'entité 1 .

Pour le contraste $1:$ les temps de lecture des phrases cibles contenant un pronom anaphorique seront plus rapides lorsque le pronom anaphorique réfère à l'entité 1 plutôt qu'à l'entité 2 . Pour référer à l'entité 1 , le pronom anaphorique sera un meilleur marqueur que la description démonstrative (TL plus rapides) alors que pour référer à l'entité 2, la description démonstrative sera un meilleur marqueur que le pronom anaphorique (TL plus rapides).

Pour le contraste 2 : aucune différence de traitement significative ne sera observée pour la description définie selon qu'elle réfère à l'entité 1 ou à l'entité 2 . Pour référer à l'entité 1 , la description définie sera un meilleur marqueur que la description démonstrative (TL plus rapides) alors que pour référer à l'entité 
2, aucune différence de traitement significative n'est attendue entre description définie et description démonstrative.

\section{Deuxième série d'études : méthodologie et approche proposée}

Dans cette deuxième série d'études, nous testerons l'hypothèse originale selon laquelle la description démonstrative (ce garçon/cette fille) constitue un bon outil référentiel pour introduire un nouveau point de vue sur un référent déjà très accessible (entité 1 ), c'est-à-dire en le présentant non pas en continuité avec le contexte précédent, mais au contraire avec un changement de perspective. Pour y parvenir, le matériel expérimental utilisé précédemment sera modifié (voir section matériel expérimental ci-dessous) de sorte que la prédication anaphorique (i.e., la phrase cible) entraîne une rupture, une 'sortie', par rapport à la situation dans laquelle le référent était impliqué.

Dans cette deuxième série d'études, trois contrastes seront utilisés :

- Le premier contraste étudiera l'opposition entre pronom anaphorique il / elle vs. description démonstrative ce garçon / cette fille. Du fait du changement de perspective introduit par la modification du matériel, aucune différence significative de traitement n'est cette fois attendue entre le pronom $\mathrm{il} / \mathrm{elle}$ et la description démonstrative pour référer à l'entité 1 . La référence à l'entité 2 sera en revanche toujours facilitée par l'utilisation de la description démonstrative (idem pour le deuxième contraste).

- Le deuxième contraste étudiera l'opposition entre description définie le garçon/la fille vs. ce garçon/cette fille. Une différence significative de traitement est cette fois attendue en faveur de la description démonstrative pour référer à l'entité 1 . Le changement de perspective devrait rendre la description définie (qui indique la continuité) particulièrement inadaptée.

- Enfin, le troisième contraste, opposant pronom démonstratif celui-ci / celle-ci et description démonstrative ce garçon / cette fille, permettra au sein même de la catégorie des démonstratifs de mettre en évidence la spécificité de la description démonstrative - mais non du pronom - pour introduire un nouveau point de vue sur l'entité 1 (différence significative de traitement). Aucune différence n'est en revanche attendue entre les deux marqueurs pour la référence à l'entité 2.

Les deux premiers contrastes seront étudiés via la tâche d'acceptabilité et la tâche de temps de lecture. Le troisième contraste inclura de plus la tâche de continuation de fragments (tâche non pertinente pour les deux autres contrastes car les marqueurs sont identiques à ceux de la première série).

\subsection{Matériel expérimental}

Les mêmes textes que précédemment seront utilisés à l'exception des phrases cibles qui seront modifiées de la façon suivante (voir tableau 2 ci-dessous) :

- 1) changement du temps verbal du passé composé (première série) au présent de l'indicatif (pour cette deuxième série) dans le but d'introduire une rupture dans l'enchaînement temporel des événements susceptible d'exprimer un nouveau point de vue sur le référent, qui, dès lors, est saisi de façon décalée par rapport au contexte précédent.

- 2) changement du type d'adverbe employé (ex : simplement remplacé par toujours) permettant de souligner le commentaire général qui est prédiqué pour chacune des deux entités. Ces modifications seront également reportées au niveau des questions (ex : Est-ce qu'Alice choisit toujours le plat le plus dispendieux ?). 
Tableau 2 : Exemple d'un texte expérimental (illustrant le premier contraste : il / elle vs. ce garçon / cette fille) selon les quatre conditions expérimentales

\begin{tabular}{|c|c|}
\hline $\begin{array}{l}\text { Phrases } 1 \text { et } 2 \\
\text { (contexte discursif) }\end{array}$ & $\begin{array}{l}\text { Au restaurant, } \text { Alice }_{(\text {entité 1) }} \text { adore prendre son temps pour lire le menu. // } \\
\text { La dernière fois, elle avait tellement hésité entre deux plats qu'elle avait } \\
\text { finalement demandé à une serveuse } \\
\text { (entité 2) } \\
\text { chose sur le menu. // }\end{array}$ \\
\hline \multicolumn{2}{|c|}{ Phrase 3 (phrase cible) : } \\
\hline Entité $1 *$ pronom & En fait, elle commande toujours le pat de jour // \\
\hline Question (oui / non) & Est-ce qu'Alice choisit toujours le plat le plus dispendieux? \\
\hline Entité $1 * \mathrm{Ce} \mathrm{N}$ & En fait, cette fille commande toujours le plat du jour // \\
\hline Question (oui/non) & Est-ce qu'Alice choisit toujours le plat le plus dispendieux? \\
\hline Entité $2 *$ pronom & En fait, elle conseille toujours le plat du jour // \\
\hline Question (oui / non) & Est-ce que la serveuse recommande toujours le plat le plus dispendieux? \\
\hline Entité $2 *$ Ce N & En fait, cette fille conseille toujours le plat du jour // \\
\hline Question (oui / non) & Est-ce que la serveuse recommande toujours le plat le plus dispendieux? \\
\hline
\end{tabular}

\subsection{Procédures utilisées dans les différentes tâches}

Pour chacun des trois contrastes de cette duexième série, les procédures utilisées pour les différentes tâches : tâche de jugement d'acceptabilité et d'identification du référent, tâche de continuation de fragments de texte, et tâche d'enregistrement de temps de lecture seront les mêmes que pour la première série.

\section{Références bibliographiques}

Ariel, M. (1990). Accessing noun-phrase antecedents, London, Routledge

Ariel, M. (1996). Referring expressions and the +/- coreference distinction. In Fretheim, T. \& Gundel, J. (Eds.). Reference and referent accessibility, Amsterdam/Philadelphia, John Benjamins, 13-35.

Ariel, M. (2006). Accessibility theory, In Brown, K. (éd.), Encyclopedia of Language and Linguistics, 14, Elsevier, pp15-18.

Brown-Schmidt, S. Byron, D. Tanenhaus. M. (2005). Beyond salience: interpretation of personal and demonstrative pronouns. Journal of Memory and Language, 53, 292-313.

Charolles, M. (1995). Comment repêcher les derniers ? Analyse des expressions anaphoriques en ce dernier. Pratiques, 85, 89-112.

Cornish, F. (1999). Anaphora, discourse and understanding. Evidence from English and French. Oxford Linguistics, Clarendon. 
Cornish, F. (2001). "Modal" that as determiner and pronoun: the primacy of the cognitive-interactive dimension. English Journal and Linguistics, 5(2), 297-315.

De Mulder, W. (1997). Les démonstratifs : des indices de changements de contexte. In Flaux N., Van de Velde D. \& De Mulder W. (Eds.) Entre général et particulier: les déterminants. Artois Presses Université, 137-200.

DeMulder (2000). Démonstratifs et accessibilité. Verbum, XXII, 1, 103-125.

Fossard, M. (1999). Traitement anaphorique et structure du discourse. Etude psycholinguistique des effets de "focus de discourse" sur la spécificité de deux marqueurs référentiels : le pronom anaphorique il et le nom propre répété. In Cognito, 15, 33-40.

Fossard, M. (2006). Aspects psycholinguistiques du traitement des démonstratifs: résultats croisés en français et en anglais. Langue Française, 152, 82-95.

Fossard, M. \& Rigalleau, F. (2005). Referential accessibility and anaphor resolution: the case of the French hybrid demonstrative pronoun celui-ci / celle-ci. In Branco, A., McEnery T \& Mitkov, R. (Eds.) Anaphora processing. Linguistic, Cognitive and Computational modelling. John Benjamins, Amsterdam/Philadelphia, 283-300.

Garnham, A. (2000). Mental models and the interpretation of anaphora. Hove : Psychology Press

Garrod, S.C., Freudenthal, D., \& Boyle, E. (1994). The role of different types of anaphor in the on-line resolution of sentences in a discourse. Journal of Memory and Language, 33, 39-68.

Gernsbacher M.A. \& Givon T. (eds). (1995). Coherence in spontaneous text. Amsterdam: John Benjamins

Gordon, P., Grosz, B., \& Gilliom, L. (1993). Pronouns, names and the centering of attention in discourse. Cognitive Science, 17, 311-347.

Greene, S.B., McKoon G., \& Ratcliff R. (1992). Pronoun resolution and discourse models. Journal of Experimental psychology: Learning, Memory and Cognition, 18(2), 266-283.

Gundel, J. (1998). Centering theory and the Givenness hierarchy:toward a synthesis. In Walker,M., Joshi, S. \& Prince, E.. (Eds.) CenteringTheory in Discourse, Oxford, Clarendon Press. 183-198

Gundel, J., Hedberg, N., \& Zacharski, R. (1993). Cognitive status and the form of referring expressions in discourse. Language, 69(2), 274-307.

Gundel, J., Hedberg, N., \& Zacharski, R. (2000). Statut cognitif et anaphoriques indirects. Verbum, XXII, 1, 79-102.

Hudson-D'Zmura, S., Tanenhaus, M.K. (1998). Assigning antecedents to ambiguous pronouns : the role of the center of attention as the default assignment. In Walker, Joshi, \& Prince. (Eds.) Centering Theory in Discourse. Oxford, Clarendon Press. 199-226.

Johnson-Laird, P.N. (1983). Mental models. Cambridge : Cambridge University Press

Kleiber, G. (1986). Déictiques, embrayeurs, ‘token-reflexives', symboles indexicaux, etc... : comment les définir ? L'Information grammaticale, 30, 3-22.

Kleiber, G. (1994). Anaphores et pronoms. Duculot, Louvain la Neuve.

Kleiber, G. (2001). Regards sur l'anaphore et la givenness hierarchy. In Langage et Référence, Acta Universitatis, 311-322.

Maes, A.A. \& Noordman, L. (1995). Demonstrative nominal anaphors: a case of nonidentificational markedness. Linguistics, 33, 255-282 
Sanford, A.J., \& Garrod, S.C. (1989). What, when and How: questions of immediacy in anaphoric reference resolution. Language and Cognitive Processes, 4, 263-287

Sanford, A.J., Moar, K., \& Garrod, S.C. (1988). Proper names as controllers of discourse focus. Language and Speech, 31, 43-56.

Strauss, S. (2002). This, That, and It in spoken American English: a demonstrative system of gradient focus. Language Sciences, 24, 131-152.

Walker, M.A., Joshi, A., \& Prince, E. (1998). Centering in naturally occurring discourse: an overview. . In Walker, Joshi, \& Prince. (Eds.) Centering Theory in Discourse. Oxford, Clarendon Press. 1-28. 